\title{
Repeated Measure Analysis of Line-source Sprinkler Experiments
}

\author{
George C.J. Fernandez ${ }^{1}$ \\ Department of Plant Science, University of Nevada-Reno, Reno, NV 89557-0107
}

Line-source sprinkler systems (LSSS), which create a continuous variable soil water gradient from the water source, have been used in irrigation (Hanks et al., 1980) and drought screening studies (ICRISAT, 1982). This system is simple to set up and minimizes land required since no border rows between plots are needed. Field plot layouts using LSSS have been published elsewhere (Hanks et al., 1980; Johnson et al., 1983).

The experimental design is similar to stripplot or split-block design (Steel and Torrie, 1980) except that irrigation levels are systematically arranged without randomization. The treatments are applied at a right angle to the irrigation gradient. Although the tests on treatments are statistically valid, no valid univariate statistical tests are available to test the main effects of irrigation and the interaction between irrigation and treatment effects (Hanks et al., 1980) unless the HuynhFeldt (H-F) condition is satisfied (Huynh and Feldt, 1970). Johnson et al. (1983) proposed multivariate methods to test the joint effects of irrigation levels and treatments. These tests are difficult to compute since complex matrix manipulations are involved.

Recently, repeated measures (RM) analysis has been recommended for experiments with data collected at several points in time or space on the same experimental units (Freund et al., 1986; Littel, 1989). The RM analysis is readily available in the Statistical Analysis System (SAS) general linear model (GLM) and analysis of variance (ANOVA) procedures (SAS, 1988). The feasibility of applying the RM analysis to the LSSS data and the comparison of the RM results with the multivariate methods of Johnson et al. (1983) are discussed in this paper.

\section{Repeated measures analysis of variance}

Agricultural researchers often conduct experiments that involve repeated measurements on the same experimental units when measurements are recorded over time or space. The experimental unit can be a plant in the greenhouse, a plot in the field, a tree in an

Received for publication 18 Aug. 1989. The cost of publishing this paper was defrayed in part by the payment of page charges. Under postal regulations, this paper therefore must be hereby marked advertisement solely to indicate this fact.

${ }^{1}$ Assistant Professor in plant breeding and biometrics. orchard, or systematically arranged plots in LSSS. Objectives of these experiments are usually to investigate the response over time or space to several treatments.

The RM experiments have been conventionally analyzed as a split-plot design taking time as the split-plot factor (Steel and Torrie, 1980). However, since the repeated measurements are systematically arranged without randomization, the repeated measure experiments are not truly split-plot designs. Thus, analysis of repeated measure experiments by a split-plot design might inflate the probability of falsely rejecting a true null hypothesis, i.e., or type I errors.

A critical assumption underlying the conventional split-plot approach to repeated measures design is that all repeated measurements have equal variances and all pairs of measurements must have the same correlation (Snedecor and Cochran, 1980). This condition is called compound symmetry and is stronger than required (Littel, 1989). A more realistic requirement is the H-F condition (Huynh and Feldt, 1970), which permits unequal variances and covariances "but does not require that the variance and covariance have a certain mathematical dependence on another set of parameters" (Littel, 1989). The statement of the mathematical condition is reported elsewhere (Milliken and Johnson, 1984). The H-F condition in RM can be verified by applying a sphericity test (Littel, 1989; SAS, 1988) to any set of transformed variables defined by a suitable orthogonal contrast transformation. When there are only two levels of repeated measurements, there is only one transformed variable and a sphericity test cannot be applied, nor is it needed (SAS, 1988).

If the RM data satisfy the H-F condition $(P>0.05)$, the $\mathrm{F}$ test in the univariate splitplot or strip-plot analysis can be used to test the RM effects and the associated interactions. If the data violate the $\mathrm{H}-\mathrm{F}$ condition, the sphericity test is significant $(P<0.05)$, a deflation of the numerator and the denominator $\mathrm{df}$ for the $\mathrm{F}$ tests involving $\mathrm{RM}$ is recommended before determining the significance levels for the univariate $\mathrm{F}$ tests (Freund et al., 1986). Two such adjustments, the Greenhouse and Geisser (1959) (G-G) epsilon and the H-F epsilon (Huynh and Feldt, 1976) are reported. Both estimate an epsilon and multiply the numerator df and the denominator $\mathrm{df}$ by the epsilon before deter- mining the significant level of the $\mathrm{F}$ test. The true value of epsilon must be between 0 and 1 (Freund et al., 1986). But H-F epsilon sometimes can be $>1$. In that case, no adjustment is needed to the $\mathrm{df}$ or to the significance levels. Huynh and Feldt (1976) have shown that the G-G adjustment is conservative and biased downward compared with the H-F adjustment, especially for small samples. If the $\mathrm{H}-\mathrm{F}$ condition is rejected at $P<0.0001$, multivariate tests (Wilk's likelihood ratio) are preferred over the adjusted univariate tests since multivariate tests do not require the H-F condition (SAS, 1988).

Repeated measure ANOVA can be performed effectively using the REPEATED statement in PROC GLM or PROC ANOVA in SAS (1988). The sphericity test is available when the PRINTE option is used with the REPEATED statement. This sphericity test is applied both to the transformed variables as defined by the REPEATED statement and to a set of orthogonal components if the specified transformation was not orthogonal. The sphericity test applied to the orthogonal components determines whether the RM data satisfy the H-F condition (SAS, 1988). Both G-G and H-F adjustments and a variety of multivariate tests are also available in SAS.

In RM analysis, a particular choice of contrast transformation provides additional insight into the data being studied. In SAS, several contrast transformations can be specified with the REPEATED statement. Neither univariate nor multivariate test statistics are affected by the type of transformation. The choice of transformation is only important in studying the nature of repeated measure effects and the associated interactions with treatments. The following transformations are available in SAS: Contrast: When one particular control level of repeated measure is compared against all other levels of the factor. Polynomial: When the levels of repeated measures represent quantitative levels and linear, quadratic effects, etc., are of specific interest to generate orthogonal polynomial contrasts. Helmert: Comparison between a level of RM and the means of all other subsequent levels. Useful to determine the point at which the response ceases to change. Mean: Each level in RM is compared to the mean of all other levels. Profile: Comparison between any two adjacent levels of RM. Useful in determining the point at 
which the response changes. The ANOVA of the transformed variables and their interactions with the treatment can be obtained by the SUMMARY option in the repeated statement. If one of these transformations does not meet the need of a particular analysis, the M option of the MANOVA statement can be used to perform the test of interest.

\section{Sample data set}

Yield data from an irrigation experiment using the LSSS and published elsewhere (Hanks et al., 1980) are used to illustrate the $\mathrm{RM}$ analysis. The original data were derived from an experimental design of three cultivars, three blocks, two north and south halves (the sprinkler line divided the experimental site into two halves), and six irrigation levels (Hanks et al., 1980). One of the requirements for computing multivariate statistics is that the numer of RM should be less than or equal to the number of experimental units per treatment combination (Johnson et al., 1983; Latour and Minard, 1983). Thus, the two north-south halves were combined with the replicates and treated as blocks. The modified data structure consists of three cultivars, six blocks, and six irrigation levels.

The RM ANOVA was performed by the PROC GLM procedure in PC-SAS. The sphericity test statistic was obtained by the PRINTE option in the REPEATED statement. Two contrast transformations, HELMERT and PROFILE, were used in RM ANOVA. The HELMERT transformation was used to determine at which irrigation level the yield response ceased to change, whereas the irrigation level at which the change took place was determined by the PROFILE transformation. A POLYNOMIAL transfor- mation was not used, since quantitative information was unavailable on the irrigation levels.

Comparison of Johnson et al.'s multivariate tests of Hank et al.'s LSSS data with RM analysis. Johnson et al. (1983) proposed multivariate tests involving complex matrix computation to analyze the LSSS data of Hanks et al. (1980). To compute valid multivariate tests, Johnson et al. (1983) modified the data of Hanks et al. by combining the north and south halves and the adjacent irrigation levels. Thus, the condensed data structure consisted of three cultivars, three blocks, and three irrigation levels (Table 1). Johnson et al. (1983) computed the multivariate test statistics, Wilk's likelihood ratio, and the probability values using matrix manipulations.

For the same condensed LSSS data, RM analysis was carried out by using the PROC GLM REPEATED statement, and multivariate test statistics were computed using the MANOVA option in PROC GLM (SAS, 1988). The M matrices (Johnson et al., 1983) were specified in the M option in PROC GLM (Table 2). Additional tests were also performed involving the contrast "Luke vs. others" and irrigation levels.

\section{Data set analysis}

The RM-ANOVA statistics, univariate, adjusted univariate, and multivariate test statistics for Hanks et al.'s original data and the condensed data are shown in Table 3. Validity of the $\mathrm{F}$ tests for the main effect of blocks (B) and cultivar (C) does not require the H-F condition of the RM. Thus, univariate tests are valid for these main factors. Main effects of cultivar across all irrigation
(IRR) levels were not statistically significant.

The sphericity test for the original data was not significant ( $\chi^{2}$ approximation 16.66 with $16 \mathrm{df} ; P>\chi^{2}=0.27$ ), indicating the correlation structure of the RM data satisfied the required $\mathrm{H}-\mathrm{F}$ conditions. The univariate, adjusted univanate, and the multivariate tests were highly significant for the IRR levels. The univariate and multivariate tests were not in agreement for the IRR $\times \mathrm{C}$ interaction. Because the univariate tests were valid for these data, the interaction between IRR $\times \mathrm{C}$ can be considered significant. The univariate test of the interaction between the contrast "Luke vs. others" and IRR was significant, indicating that the yield of "Luke" was greater than the other cultivars under high irrigation levels and vice versa under low irrigation levels. (Fig. 1) These results were in agreement with the Hanks et al. (1980) results. However, the RM analysis provided valid statistical tests for the main effect of irrigation and the associated interactions.

RM analysis for the condensed data. Combining the adjacent irrigation levels and the north and south halves did not change the statistical significance of the main effects of B and C (Table 3). However, the sphericity test results $\left(\chi^{2}\right.$ approximation of 7.75 with $\left.2 \mathrm{df} ; P>\chi^{2}=0.0207\right)$ indicated that the condensed data violated the H-F condition. Thus, the univariate tests involving RM and the associated interactions might result in an inflated Type I error. The MANOVA tests that do not require the H-F condition (Littel, 1989) or the adjusted univariate tests by the G-G epsilon (0.511) or the H-F epsilon (1.24) can be used to test the RM and the associated interactions. Because the $\mathrm{H}-\mathrm{F}$ epsilon was $>1$ no adjustment was made to the univariate significance levels (Table 3).

Table 1. Condensed wheat yield data (Johnson et al., 1983) obtained from the line-source sprinkler experiment of Hanks et al. (1980).

\begin{tabular}{|c|c|c|c|c|c|c|c|c|c|}
\hline \multirow[b]{4}{*}{ Block } & \multicolumn{9}{|c|}{ Cultivars } \\
\hline & \multicolumn{3}{|c|}{ Bridger } & \multicolumn{3}{|c|}{ Luke } & \multicolumn{3}{|c|}{ Nugaines } \\
\hline & \multicolumn{9}{|c|}{ Irrigation levels } \\
\hline & $\mathrm{IRR}_{1}$ & $\mathrm{IRR}_{2}$ & $I_{R R_{3}}$ & $\mathrm{IRR}_{1}$ & $\mathrm{IRR}_{2}$ & $\mathrm{IRR}_{3}$ & $\mathrm{IRR}_{1}$ & $\mathrm{IRR}_{2}$ & $\mathrm{IRR}_{3}$ \\
\hline I & 13.3 & 23.9 & 25.7 & 10.7 & 27.2 & 28.4 & 13.0 & 21.1 & 26.5 \\
\hline II & 12.6 & 25.2 & 27.0 & 9.9 & 23.7 & 28.6 & 10.8 & 22.9 & 25.7 \\
\hline III & 11.7 & 18.9 & 22.4 & 9.4 & 19.8 & 24.8 & 10.4 & 19.3 & $21.2^{z}$ \\
\hline
\end{tabular}

$z^{2}$ This number was corrected to obtain the analysis given by Johnson et al. (1983).

Table 2. The SAS program used to perform multivariate analyses of the condensed LSSS data (Johnson et al., 1983).

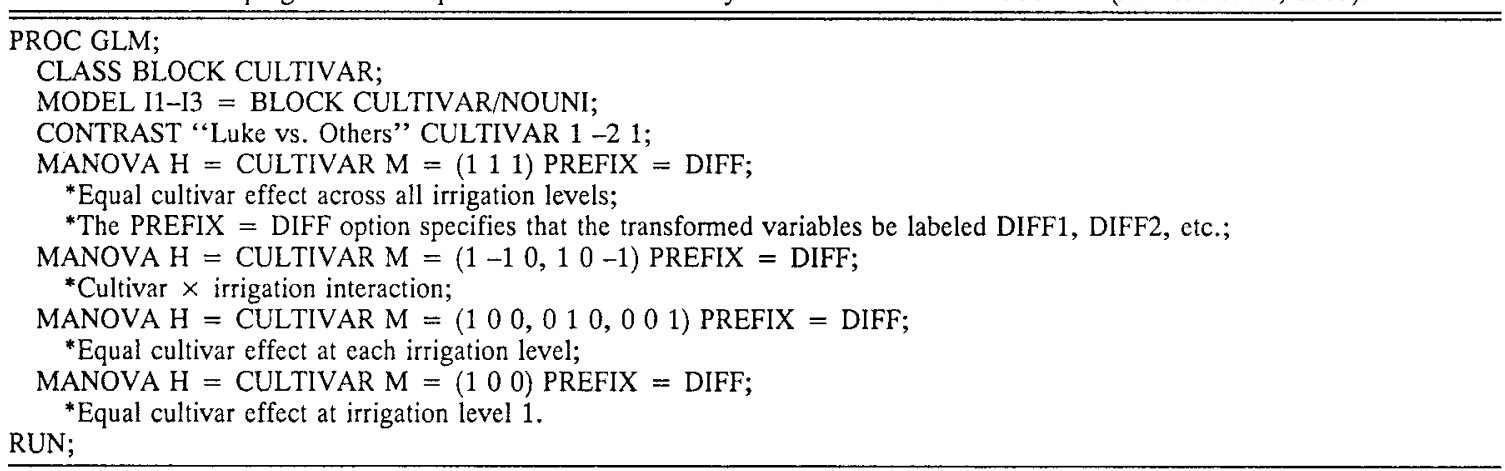


However, the results of the multivariate tests and the adjusted univariate test were not in agreement for IRR $\times \mathrm{B}$ and IRR $\times \mathrm{C}$. The IRR $\times C$ interaction was significant in the MANOVA test; not significant in the G-G adjusted test $(P>\mathrm{F} 0$. 104); and significant in the $\mathrm{H}-\mathrm{F}$ adjusted test $(P>\mathrm{F} 0.043)$. These results clearly confirmed that the G-G adjustment was more conservative than the $\mathrm{H}-\mathrm{F}$ adjustment. When the H-F condition is met in RM analysis, Littel (1989) suggested using the G-G adjusted probability as a compromise between the univariate and multivariate tests when univariate probability values cannot be trusted and multivariate tests are not significant. Latour and Minard (1983) recommended multivariate tests over G-G and $\mathrm{H}-\mathrm{F}$ adjusted univariate tests if one is concerned about the Type I errors. When the $\mathrm{H}-\mathrm{F}$ condition is not met, Huynh and Feldt (1976) showed that the G-G adjustment is more conservative and has the disadvantage of underestimating the true significance levels. Therefore, the following recommendations are made regarding testing RM effects: 1) If the H-F condition is met (the sphericity test is not significant) the univariate test statistics can be used. 2) If the H-F condition

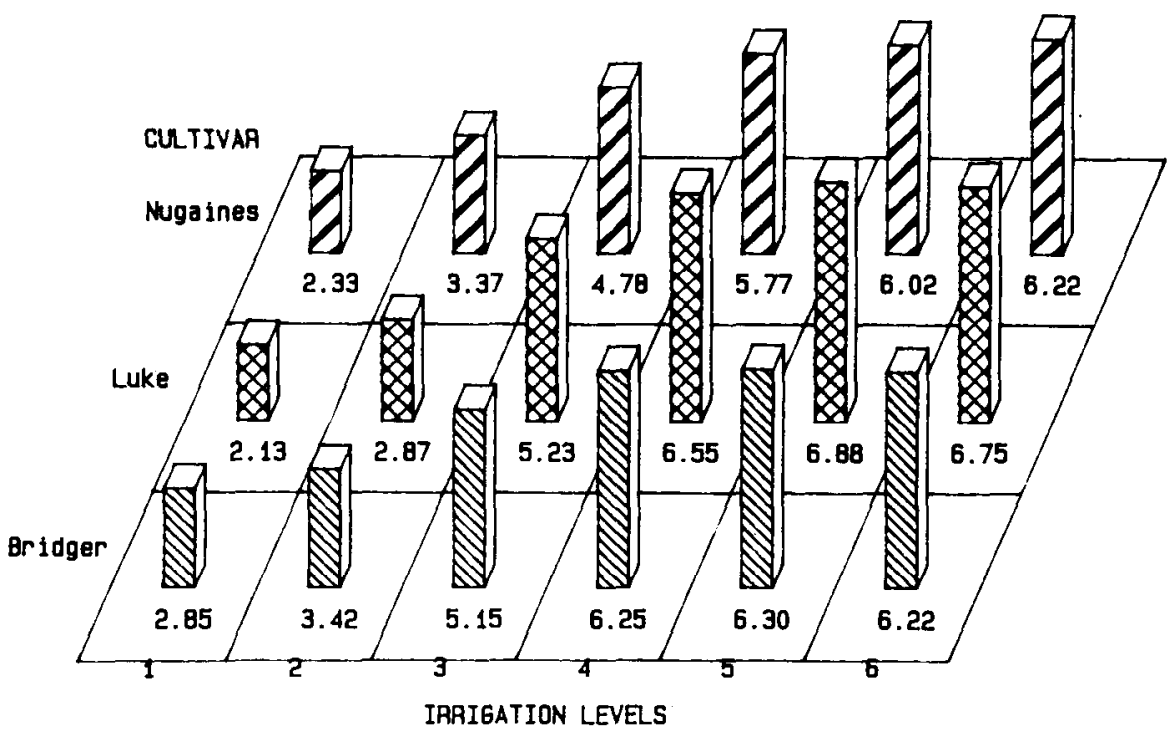

Fig. 1. Cultivar $\times$ irrigation interaction of Hanks et al.'s line-source sprinkler system data.

is violated and the sphericity test is significant at the 0.05-0.01 level, either the H-F adjusted univariate tests or multivariate tests can be used. 3) If the H-F condition is rejected at $<0.01$ level, only the multivariate tests should be used.

$R M$ analysis on the transformed variables. The RM analysis in SAS also provides additional information on the RM data by performing ANOVA on contrast variables involving the RM. The ANOVA statistics on contrast variables transformed by the HELMERT $\left(\operatorname{IRR}_{\mathrm{h}}\right)$ and PROFILE transformation (IRR.) are shown in Table 4. The Helmert transformation was used to test the effect of one irrigation level vs. the mean of the subsequent levels, whereas the effects of the adjacent levels were tested by the profile transformation. Both contrast transformations indicated that the average effects of irrigation levels $I R R_{1}, I R R_{2}$, and $I_{R R}$ were significantly different. The interactions between cultivar and the contrasts of $\mathrm{IRR}_{1}$ vs. subsequent levels $\left(\operatorname{IRR}_{\mathrm{In}}\right)$, and $\mathrm{IRR}_{2} \mathrm{vs}$. subsequent levels $\left(\operatorname{IRR}_{2 \mathrm{~h}}\right)$ were significant, whereas the interaction of cultivar $\times$ the contrast of $\mathrm{IRR}_{3}$ vs. subsequent levels $\left(\mathrm{IRR}_{3 \mathrm{~h}}\right)$ was not significant. These results indicated that cultivars responded differently in IRR and $I_{2} R_{2}$ than at other irrigation levels. The interactions between the contrast "Luke vs. others" $\times \operatorname{IRR}_{\mathrm{lh}}$ and $\mathrm{IRR}_{2 \mathrm{~h}}$ were highly significant, indicating that 'Luke' responded differently than the other two cultivars at IRR and $\mathrm{IRR}_{2}$. This interaction is shown in Fig. 1.

The ANOVA statistics for the contrast variable obtained by the profile transforma-

Table 3. A comparison of ANOVA for the original and the condensed data obtained from Hanks et al. (1980) in a line-source sprinkler experiment by RM analysis.

\begin{tabular}{|c|c|c|c|c|c|c|c|c|c|c|c|}
\hline \multirow[b]{2}{*}{ Source } & \multirow[b]{2}{*}{$\mathrm{df}$} & \multirow[b]{2}{*}{ SS } & \multicolumn{2}{|c|}{$\begin{array}{c}\text { Univariate } \\
\text { analysis }\end{array}$} & \multicolumn{2}{|c|}{$\begin{array}{l}\text { Adjusted } \\
\text { univariate }\end{array}$} & \multicolumn{5}{|c|}{ MANOVA tests } \\
\hline & & & F value & $P>\mathrm{F}$ & $G-G^{2}$ & $\mathrm{H}-\mathrm{F}^{y}$ & Lambdax & F value & $\mathrm{N}-\mathrm{df} \mathrm{f}^{\mathrm{w}}$ & $\mathrm{D}-\mathrm{d} \mathrm{f}^{\mathrm{v}}$ & $P>\mathrm{F}$ \\
\hline & \multicolumn{11}{|c|}{ Hanks et al. data } \\
\hline Blocks (B) & 5 & 21.00 & 13.52 & 0.0004 & --- & --. & $\cdots$ & -.. & $\ldots$ & -.- & ... \\
\hline Cultivar (C) & 2 & 2.16 & 3.48 & 0.074 & ... & ... & ..- & ..- & .-. & $\ldots$ & ... \\
\hline "Luke vs. Others" & 1 & 0.83 & 2.68 & 0.13 & .-- & ... & ... & ..- & $\ldots$ & $\ldots$ & ... \\
\hline $\mathrm{B} \times \mathrm{C}($ Error-a) & 10 & 3.10 & $\ldots$ & --- & ...- & ... & $\ldots$ & ... & ... & .-- & $\ldots$ \\
\hline Irrigation (IRR) & 5 & 269.8 & 182.1 & 0.0001 & 0.0001 & 0.0001 & 0.0069 & 170.2 & 5 & 6 & 0.0001 \\
\hline $\mathrm{IRR} \times \mathrm{B}$ & 25 & 20.6 & 2.7 & 0.0010 & 0.0083 & 0.0010 & 0.0151 & 1.9 & 25 & 24 & 0.049 \\
\hline $\mathrm{IRR} \times \mathrm{C}$ & 10 & 6.6 & 2.2 & 0.031 & 0.068 & 0.031 & 0.182 & 1.6 & 10 & 12 & 0.216 \\
\hline IRR $\times$ "Luke vs, others" & 5 & 5.9 & 4.8 & 0.0038 & 0.016 & 0.0038 & 0.298 & 2.8 & 5 & 6 & 0.119 \\
\hline $\mathrm{B} \times \mathrm{C} \times \operatorname{IRR}($ Error-b) & 50 & 14.8 & ... & -.- & $\ldots$ & -... & $\ldots$ & $\ldots$ & $\ldots$ & ... & $\ldots$ \\
\hline \multicolumn{12}{|c|}{ Sphericity test: $x^{2}$ approximation 16.66 with $14 \mathrm{df} ; P>\chi^{2}=0.2747^{\mathrm{NS}}$} \\
\hline \multicolumn{12}{|c|}{ G } \\
\hline Blocks (B) & 2 & 68.20 & 40.69 & 0.002 & --- & $\cdots$ & --- & ... & ... & --- & -.- \\
\hline Cultivar (C) & 2 & 8.66 & 5.17 & 0.077 & -- & $\cdots$ & -.. &.-- & $\cdots$ & -.. & ... \\
\hline "Luke vs. others" & 1 & 3.32 & 3.97 & 0.117 & ב.- & $\ldots$ & ... & $\ldots$ & $\ldots$ & $\ldots$ & $\ldots$ \\
\hline$B \times C$ (Error-a) & 4 & 3.55 & --- & -.. & ... & $\ldots$ & ... & ... & ... & -.. & ...- \\
\hline Irrigation (IRR) & 2 & 1013 & 351.1 & 0.0001 & 0.0001 & 0.0001 & 0.0002 & 6256 & 2 & 3 & 0.0001 \\
\hline $\mathrm{IRR} \times \mathrm{B}$ & 4 & 15.7 & 2.7 & 0.106 & 0.175 & 0.106 & 0.0198 & 9.1 & 4 & 6 & 0.01 \\
\hline $\mathrm{IRR} \times \mathrm{C}$ & 4 & 23.4 & 4.0 & 0.043 & 0.104 & 0.043 & 0.0109 & 13.5 & 4 & 6 & 0.0037 \\
\hline IRR $\times$ "Luke vs. others" & 2 & 22.7 & 7.8 & 0.012 & 0.045 & 0.012 & 0.010 & 137.2 & 2 & 3 & 0.001 \\
\hline $\mathrm{B} \times \mathrm{C} \times \mathrm{IRR}$ & 8 & 11.5 & 1.4 & -.. & -..- & $\ldots$ & ... & $\ldots$ & $\ldots$ & ... & ... \\
\hline \multicolumn{12}{|c|}{ Sphericity test: $\chi^{2}$ approximation 7.75 with $2 \mathrm{df} ; P>\chi^{2}=0.0207^{*}$} \\
\hline
\end{tabular}

${ }^{2} \mathrm{G}-\mathrm{G}=$ The adjusted probability value by the Greenhouse-Geiser epsilon $=0.598$ for Hank's data and 0.511 for the condensed data.

$\mathrm{y}_{\mathrm{H}-\mathrm{F}}=$ The adjusted probability by the Huynh-Feldt epsilon $=1.48$ for Hank's data and 1.24 for the condensed data.

${ }^{x}$ Lambda $=$ Wilk's Lambda.

"N-df $=$ Numerator $\mathrm{df}$.

${ }^{\mathrm{D}} \mathrm{D}-\mathrm{df}=$ Denominator degrees of freedom. 
Table 4. A comparison of repeated measures ANOVA for the contrast variables $\left(\mathrm{IRR}_{1}-\mathrm{IRR}_{3}\right)$ associated with four irrigation levels in a line-source sprinkler system experiment.

\begin{tabular}{|c|c|c|c|c|c|c|c|c|}
\hline \multirow[b]{2}{*}{ Source } & \multicolumn{4}{|c|}{ Helmert transformation } & \multicolumn{4}{|c|}{ Profile tranformation } \\
\hline & $\mathrm{df}$ & SS & F value & $P>\mathrm{F}$ & df & SS & F value & $P>\mathrm{F}$ \\
\hline \multicolumn{5}{|c|}{$I R R_{I h}:$ Mean of $I R R_{1} v s$. mean of subsequent levels } & \multicolumn{4}{|c|}{$I R R_{I p} \cdot$ Means of IRR $R_{1}$ vs. IRR } \\
\hline $\mathrm{IRR}_{1}$ & 1 & 162.8 & 815.9 & 0.0001 & 1 & 10.88 & 46.23 & 0.0001 \\
\hline $\mathrm{IRR}_{1} \times$ blocks & 5 & 4.2 & 4.3 & 0.02 & 5 & 6.72 & 5.71 & 0.009 \\
\hline $\mathrm{IRR}_{1} \times$ cultivars & 2 & 2.7 & 6.6 & 0.01 & 2 & 0.67 & 0.08 & 0.28 \\
\hline $\mathrm{IRR}_{1} \times$ "Luke vs. others" & 1 & 2.4 & 11.9 & 0.0061 & 1 & 0.01 & 0.08 & 0.78 \\
\hline Error & 10 & 1.99 & $\cdots$ & $\cdots$ & 10 & 2.35 & $\cdots$ &.- \\
\hline \multicolumn{5}{|c|}{$I R R_{2 h}:$ Mean of $I R R_{2}$ vs. mean of subsequent levels } & \multicolumn{4}{|c|}{$I R R_{2 p} \cdot$ Means of $I R R_{2}$ vs. $I R R_{3}$} \\
\hline $\mathrm{IRR}_{2}$ & 1 & 139.4 & 316.8 & 0.0001 & 1 & 60.86 & 122.1 & 0.0001 \\
\hline $\mathrm{IRR}_{2} \times$ blocks & 5 & 9.2 & 4.2 & 0.02 & 5 & 5.72 & 2.3 & 0.123 \\
\hline $\mathrm{IRR}_{2} \times$ cultivars & 2 & 4.5 & 5.2 & 0.02 & 2 & 2.80 & 2.8 & 0.10 \\
\hline $\mathrm{IRR}_{2} \times$ “Luke vs. others" & 1 & 4.4 & 10.0 & 0.0100 & 1 & 2.50 & 5.03 & 0.04 \\
\hline Error & 10 & 4.41 & $\cdots$ & $\cdots$ & 10 & 4.08 & $\cdots$ & $\cdots$ \\
\hline \multicolumn{5}{|c|}{$I R R_{3 h}:$ Mean of $I R R_{3}$ vs. mean of subsequent levels } & \multicolumn{4}{|c|}{$I R R_{3 p} \cdot$ Means of $I R R_{3}$ vs. IRR } \\
\hline $\mathrm{IRR}_{3}$ & 1 & 28.7 & 70.8 & 0.0001 & 1 & 22.21 & 48.1 & 0.0001 \\
\hline $\mathrm{IRR}_{3} \times$ blocks & 5 & 10.8 & 5.3 & 0.01 & 5 & 7.93 & 3.43 & 0.04 \\
\hline $\mathrm{IRR}_{3} \times$ cultivar & 2 & 0.5 & 0.6 & 0.54 & 2 & 0.38 & 0.19 & 0.66 \\
\hline $\mathrm{IRR}_{3} \times$ "Luke vs. others" & 1 & 0.5 & 1.2 & 0.30 & 1 & 0.38 & 0.82 & 0.38 \\
\hline Error & 10 & 4.06 & -.. & $\ldots$ & 10 & 4.21 & $\cdots$ & $\cdots$ \\
\hline
\end{tabular}

Table 5. Analysis of variance of the condensed data (Johnson et al., 1983) obtained in a line-source sprinkler system experiment by multivariate (MANOVA) techniques.

\begin{tabular}{lccllc}
\hline \hline Source & N-dfz & D-dfy & Lambda & F value & $P>\mathrm{F}$ \\
\hline Equal cultivar effect across all irrigation levels & 2 & 4 & 0.279 & 5.17 & 0.077 \\
"Luke vs. other" across all irrigation levels & 1 & 4 & 0.502 & 3.967 & 0.112 \\
Cultivar X irrigation interaction & 4 & 6 & 0.009 & 13.5 & 0.0037 \\
"Luke vs. Other" x irrigation interaction. & 2 & 3 & 0.0108 & 137.2 & 0.0011 \\
Equal cultivar effect at each irrigation level & 6 & 4 & 0.00154 & 16.3 & 0.0088 \\
"Luke vs. other" at each irrigation level & 3 & 2 & 0.00614 & 107.8 & 0.0092 \\
Equal cultivar effect at irrigation level-1 & 2 & 4 & 0.078 & 23.43 & 0.0062 \\
"Luke vs. other" at irrigation level-1 & 1 & 4 & 0.096 & 37.53 & 0.0036 \\
\hline
\end{tabular}

${ }^{2} \mathrm{~N}-\mathrm{df}=$ numerator degrees of freedom.

${ }^{y} \mathrm{D} \cdot \mathrm{df}=$ denominator degrees of freedom.

'Wilk's Lambda.

tion indicated the IRR level at which the response change occurred. The interaction between the contrast "Luke vs. others" $x$ the contrast of IRR VS. IRR $_{2}\left(\operatorname{IRR}_{1 \mathrm{p}}\right)$ was not significant, whereas the "Luke vs. others" $\times$ the contrast of $\operatorname{IRR}_{2}$ vs. $\operatorname{IRR}_{3}\left(\operatorname{IRR}_{2 \mathrm{p}}\right)$ was significant, which indicated the response change occurred between $\mathrm{IRR}_{2}$ and $\mathrm{IRR}_{3}$. Thus, these contrast transformations revealed additional insight into the $\mathrm{RM}$ effects.

Comparison between Johnson et al.'s multivariate tests and RM analysis. The multivariate tests suggested by Johnson et al. (1983) using a matrix procedure can be easily obtained by using the MANOVA and M options. The same statistics were obtained in MANOVA and Johnson et al.'s method (Table 5). The equality of cultivar effects across all IRR levels and $\mathrm{C} \times$ IRR interaction can be easily tested by the univariate and RM analysis, respectively. If special tests such as "equal cultivar effect at each irrigation level" and "equal cultivar effect at IRR" are necessary, the MANOVA and M options can be used effectively instead of using complex matrix computations. Furthermore, RM analysis is more powerful since it tests the $\mathrm{H}-\mathrm{F}$ condition and provides univariate, adjusted univariate, and multivariate tests.

In conclusion, RM analysis is recommended for the analysis of data obtained from experiments with repeated measurements either over time or space. Univariate analysis is suitable if the H-F condition is valid. If the $\mathrm{H}-\mathrm{F}$ condition is not met, adjusted univariate analysis or multivariate tests should be used.

\section{Literature Cited}

Freund, R.J., R.C. Littel, and P.C. Spector. 1986. SAS system for linear models. A guide to the ANOVA and GLM procedures. SAS Institute, Inc., Cary, N.C.

Greenhouse, S.W. and S. Geisser. 1959. On methods in the analysis of profile data. Psychometrika 32:95-112.

Hanks, R.J., D.V. Sisson, R.L. Hurst, and K.G. Hubbard. 1980. Statistical analysis of results from irrigation experiments using the line-source sprinkler system. Soil Sci. Soc. Amer. J. 44:886888.

Huynh, H. and L.S. Feldt. 1970. Conditions under which mean square ratios in repeated measurement designs have exact F-distribution. J.
Amer. Stat. Assn. 65:1582-1589.

Huynh, H. and L.S. Feldt. 1976. Estimation of the Box correction for degrees of freedom from sample data in randomized block and split-plot design. J. Educ. Stat. 1:69-82.

ICRISAT, 1982. International Crops Research Institute for Semi Arid Tropics. Annu. Rpt. 1981. Patancheru, A.P. India.

Johnson, D.E., U.N. Chaudhuri, and E.T. Kanemasu. 1983. Statistical analysis of line source sprinkler experiments and other nonrandomized experiments using multivariate methods. Soil Sci. Soc. Amer. J. 47:309-312.

Latour, S.A. and P.W. Minard. 1983. The misuse of repeated measure analysis in marketing research. J. Marketing Res. 20:45-57.

Littel, R.C. 1989. Statistical analysis of experiments with repeated measurements. HortScience 24:37-40.

Milliken, G.A. and D.E. Johnson. 1984. Analysis of messy data. Van Nostrand Reinhold, New York.

SAS Institute, Inc. 1988. SAS/STAT user's guide, release 6.03. SAS Institute Inc., Cary, N.C.

Snedecor, G.W. and W.G. Cochran. 1980. Statistical methods. 7th ed. Iowa State Univ. Press, Ames, Iowa.

Steel, R.G.D. and J.H. Torrie. 1980. Principles and procedures of statistics. 2nd ed. McGraw Hill, New York. 